\title{
FACTORES DE ÉXITO EN PROVEEDORES DE BIENES MANUFACTURADOS DE LA SALMONICULTURA CHILENA
}

\section{SUCCESS FACTORS IN SUPPLIERS OF MANUFACTURES WITHIN THE CHILEAN SALMON INDUSTRY}

\author{
Carlos Torres Fuchslocher ${ }^{* 1} \quad$ Hanns de la Fuente Mella ${ }^{2}$ \\ Recibido 15 de junio de 2007, aceptado 14 de noviembre de 2008 \\ Received: June 15, 2007 Accepted: November 14, 2008
}

\begin{abstract}
RESUMEN
Este artículo analiza, en base a un trabajo empírico, los principales factores a nivel de la empresa que han determinado el éxito de los proveedores locales de manufacturas, en términos de participación de mercado, en el cluster del salmón en Chile. La investigación envolvió un levantamiento de material empírico a través de 50 entrevistas, las cuales fueron evaluadas cualitativamente y por medio de un análisis econométrico de corte transversal, usando variables explicativas cualitativas. Los resultados destacan la especialización sectorial, la innovación en productos especializados para la industria y la cooperación horizontal, dentro de la estrategia de entrada; y la antigüedad de la empresa, la capacidad de innovación interna, la capacidad financiera (capacidad de otorgar crédito a clientes y bajo nivel de endeudamiento) y la diversificación de la cartera productos, dentro de la competitividad previa, como determinantes del éxito de los proveedores.
\end{abstract}

Palabras clave: Desarrollo de proveedores, tecnología, salmón, Chile.

\begin{abstract}
This article analyses the main factors at the firm-level which have determined the success, in terms of market share, of manufacture suppliers within the Chilean salmon industry. The analysis is based on data collected in an empirical research that included 50 interviews with suppliers. The data was evaluated through both a qualitative analysis and a cross section econometric analysis. The results show the importance of sectoral specialization, the innovation on specialized products for the aquaculture industry and horizontal cooperation within the entry strategy of firms, whereas the firm's age, the internal innovation capabilities, the own capital and the diversification of product portfolio were the main competitive factors before the entry.
\end{abstract}

Keywords: Development of suppliers, technology, salmon industry, Chile.

\section{INTRODUCCIÓN}

El estudio de clusters ha sido abordado desde diversas corrientes en los últimos años. Entre estas corrientes se pueden mencionar el concepto de especialización flexible [18], el diamante de Porter [19], la competitividad sistémica [6], el aprendizaje localizado [15], la generación económica local [20], la eficiencia colectiva [25]. El resurgimiento del concepto de distrito industrial ha confirmado las afirmaciones expuestas por [14] referentes a que la concentración geográfica de empresas genera ventajas para el conjunto, llamadas economías externas, y que provienen, entre otras, de la concentración de mano de obra especializada, derrame tecnológico y presencia de proveedores $([14,11,19])$.

Dentro de los proveedores de un cluster, un artículo reciente [31] distingue dos tipos: intensivos en el uso de mano de obra (proveedores de servicios que no requieren mano de obra calificada y proveedores de bienes no diferenciados) e intensivos en el uso de tecnología o proveedores-t (proveedores de productos o servicios que requieren

\footnotetext{
1 Universidad de Talca. Facultad de Ingeniería. Camino Los Niches km 1 s/n. Curicó, Chile. E-mail: catorres@utalca.cl

2 Universidad de Talca. Facultad de Ingeniería. Camino Los Niches km 1 s/n. Curicó, Chile. E-mail: hdelafuente@utalca.cl

* Correspondencia a Carlos Torres Fuchslocher. Facultad de Ingeniería. Universidad de Talca. Merced 437. Curicó, Chile. F: +56-75-201779, Fax: $+56-75-325958$
} 
capacidades especiales tales como conocimiento, capital y acceso a tecnologías). Los últimos son actores importantes para asegurar la competitividad de las industrias que atienden, lo cual queda de manifiesto en diversos estudios en Europa, Canadá y Australia ([1, 8, 19, 21, 13]).

El cluster chileno del salmón ha sostenido un dinámico desarrollo, lo que la ha convertido en el cuarto sector exportador a nivel nacional, alcanzando un total de ventas de 1.439 millones de dólares en el año 2004 [23]. Esto ha impulsado una alta demanda por diversos bienes y servicio estimulando el desarrollo de proveedores locales. Dentro de los proveedores-t se encuentran proveedores de: vacunas, ingeniería, consultoría especializada, maquinaria, sistemas de tratamiento de aguas, sistemas de control, insumos químicos y biotecnológicos, y otros tipos de manufacturas. Tal como se muestra en la figura 1 , además de los proveedores-t se han desarrollado otros proveedores intensivos en el uso de mano de obra y que dependen exclusivamente de las actividades de la cadena productiva central [31].

Los proveedores de manufacturas locales han llegado a cubrir el 50\% de la demanda de la industria, sin embargo, se observan diferencias respecto a su participación en las distintas etapas del proceso productivo y en el nivel de tecnología de los productos suministrados, donde su participación en productos más sofisticados es limitada respecto a sus contrapartes extranjeras [31].

Si bien es cierto, el éxito en el desarrollo de proveedores-t depende de factores asociados al potencial que ofrece la industria salmonicultora para la inserción de proveedores locales (tales como el tamaño y crecimiento de demanda, la estructura de la industria y la concentración geográfica), también depende de factores internos de los proveedores o potencial endógeno de los proveedores determinado por sus recursos y capacidades dinámicas [31]. Ahora bien, cabe preguntarse ¿cuáles han sido los principales factores internos que han determinado el éxito de proveedores- $t$ locales?

\section{CONTEXTO TEÓRICO}

\section{Proveedores en clusters}

La existencia de complementariedades dinámicas induce a los distritos industriales a tener un mejor desempeño que la suma de sus unidades individuales. La conformación de distritos industriales o "clusters" ha probado ser benéfica dado que se ha reconocido que la competitividad es de origen sistémico y la evolución de redes genera un potencial de acción colectiva ([14], [25, 6]). La concentración geográfica de empresas responde, en esencia, a tres factores básicos: ganancias crecientes a nivel de la empresa (economías internas) y a nivel de la industria (economías externas), costos de transporte bajos y una demanda local importante [11]. Sin embargo, la disponibilidad de factores, tales como la proximidad a una fuente de insumos de producción, bajos costos de energía y materias primas en el caso de industrias basadas en recursos naturales, han sido y continúan siendo determinantes principales para la localización de industrias en lugares particulares.

Los clusters poseen lo que se llama eficiencia colectiva, la que está compuesta por dos factores: uno de carácter pasivo, llamado externalidades o economías externas y otro de carácter activo, llamado acción colectiva. Las externalidades son el resultado del simple hecho de existir una concentración de empresas relacionadas entre sí, por ejemplo, las empresas se pueden especializar en alguna actividad y por lo tanto, pese a que sean pequeñas, en conjunto podrían lograr economías de escala comparables con las de una empresa grande integrada verticalmente, pero con mayor flexibilidad que estas últimas. Otras externalidades positivas relevantes son la disminución de los costos de transporte, la transferencia de información y tecnología (llamado derrame tecnológico), el desarrollo de capacidades y especialización de la mano de obra local y la proliferación de proveedores y servicios relacionados ([14], [11]).

Por otra parte la acción colectiva es un acto voluntario, activo y consciente de cooperación en beneficio del conjunto, es decir en clusters funciona simultáneamente la competencia y la cooperación, por ejemplo, un grupo de exportadores forma una asociación que los represente internacionalmente para la promoción de sus productos y para su defensa ante acusaciones de dumping. Sin embargo, también existe acción colectiva a través de redes informales.

En referencia a los proveedores, Marshall escribió que “...el comercio subsidiario crecen en la vecindad [de distritos industriales], proveyéndolo de implementos y materiales, organizando su tráfico y, en diferentes maneras, conduciendo a la economía de materiales" [14]. Autores más recientes, enfatizan aún más en la ventaja de proveedores locales. Proveedores participan activamente en el desarrollo del conocimiento colectivo del cluster, son fuentes de innovación y promueven la transferencia de tecnología [4]. En particular, la transferencia de conocimiento tácito inserto en bienes y servicios se ha transformado en una vía efectiva de diseminación del conocimiento base [15]. 


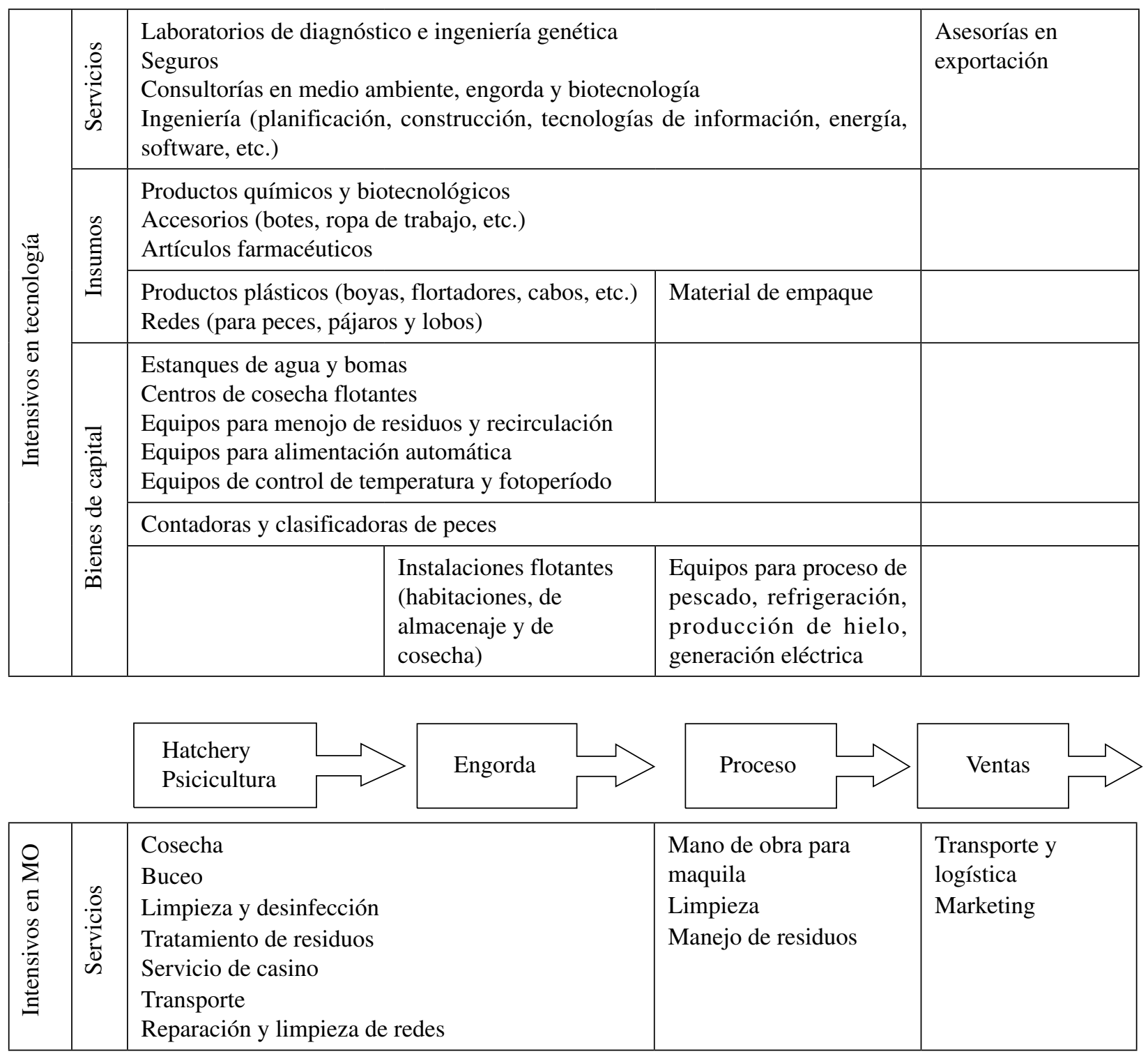

Fuente: [31].

Figura 1. Proveedores de la industria chilena del salmón.

Porter desarrolló un modelo de competitividad que intenta explicar el éxito de ciertos países en ciertas industrias [19]. Este modelo ha sido llamado Diamante de Porter, de acuerdo a la definición del autor, la cual presenta cuatro determinantes de la competitividad puestos en forma de diamante. Estos determinantes son: condiciones de los factores; condiciones de la demanda; estrategias empresariales, estructura y rivalidad; e industrias relacionadas y de soporte. Estos cuatro factores interactúan constantemente y son influenciados por factores externos tales como el gobierno (a través de sus diversas políticas) y hechos fortuitos.
Porter usa el término industrias de soporte en vez de proveedores para enfatizar el rol de soporte de la competitividad que impulsa la presencia de proveedores locales en la competencia de las industrias de un país. Proveedores internacionales competitivos sustentan la competitividad de los productores de máquinasherramienta japonesas, los productores de acero suecos y los productores de calzado italianos. Por el contrario, el bajo desarrollo relativo de Alemania y Suecia en bienes de consumo intensivos en marketing se explica en parte por la falta de competitividad internacional de sus respectivos sectores de media [19]. 
La fuente de competitividad impuesta por los proveedores puede ser explicada de diversas maneras. Los proveedores se encargan de ofrecer insumos en forma eficiente, temprana y rápida, además de ofrecer la posibilidad de coordinación, reducción de costos de transacción, costos de inventario y riesgo. Adicionalmente, los proveedores locales sustentan el proceso de innovación y upgrading. Los proveedores ayudan a las empresas a descubrir nuevos métodos y oportunidades para aplicar nuevas tecnologías. Los proveedores locales también permiten una mayor cooperación en I+D y una transferencia de información más fluida y promueven el desarrollo de soluciones eficientes y oportunas. Sin embargo, la obtención de los beneficios asociados a la presencia de proveedores locales no ocurre automáticamente. Aunque la proximidad geográfica y cultural facilita el flujo de información, las empresas deben cooperar y estar dispuestas a aprender de las demás $([4,19])$.

\section{Proveedores en cadenas de valor}

Otro enfoque es el dado por el análisis de la cadena de valor, entendiéndose como "el conjunto de actividades de un proceso productivo desde el diseño o concepción del producto hasta la entrega al consumidor final y eventualmente su reutilización" [10]. Generalmente la literatura enfatiza estos encadenamientos a través de las fronteras entre empresas productivas y sistemas de distribución, lo cual permite apreciar todo el ciclo (diseño del producto - recepción por parte del consumidor final) como un proceso de interacción continua y de agregación de valor.

Las empresas proveedoras están conectadas con la industria a la cual proveen o industria principal, que corresponde a aquellas actividades situadas en el eje central de la cadena. Por una parte, los proveedores sustentan la competitividad de las empresas a quienes proveen (a través de precios e innovaciones), crean puestos de trabajo, potencian la intercambiabilidad de productos con otras industrias, contribuyen a la diversificación de la mano de obra y de la economía en enclaves industriales, y aseguran la continuidad tecnológica entre las procesadoras de recursos naturales y el resto de la industria local.

Por otra parte, los proveedores son por lo general pequeñas y medianas empresas (PyMEs), por lo cual su desarrollo está limitado por las dificultades propias de este tipo de empresas. Además, por el hecho de no pertenecer al eje central de la cadena del valor su vínculo con el desarrollo de la industria no está garantizado. De hecho, los encadenamientos entre proveedores y clientes en cadenas de valor son caracterizados por diferentes grados de intensidad. Mientras que los proveedores que hacen uso intensivo de mano de obra de baja calificación tienden a ser totalmente dependientes de la industria demandante y están localizados cerca de ella, los proveedores de insumos, bienes de capital y servicios especializados e intensivos en conocimiento necesitan más que el simple efecto de encadenamiento de una industria. Esto significa, que la demanda de una industria, cuyo efecto de encadenamiento no es suficiente para la creación o instalación de nuevos proveedores tecnológicos locales, será suplida por importaciones o bien por la reorientación de proveedores de otros sectores de niveles tecnológicos compatibles. Estos proveedores atienden, por lo general, varias industrias, pero el vínculo con cada una de ellas es débil.

\section{Factores internos de los proveedores-t}

Torres concluye que el potencial de inclusión de proveedores-t depende de dos tipos de factores: "El primero se refiere a factores asociados al potencial que ofrece la industria salmonicultora para la inserción de proveedores locales y considera: el tamaño y crecimiento de demanda, la estructura de la industria y la concentración geográfica. El segundo se refiere a factores internos de los proveedores, la competitividad previa a la entrada como proveedor y su estrategia de inserción" [31].

Dentro del segundo grupo de factores el autor hace referencia al enfoque basado en los recursos (resource-based view) para concluir que las competencias adquiridas se refieren a capacidades o recursos internos, resultantes de la historia de la empresa y que, a su vez, están asociados a las áreas funcionales de la empresa (operaciones, gestión, finanzas y marketing), junto a otros factores intrínsecos o endógenos ([17, 32]). Mientras que para establecer los factores involucrados en la estrategia de entrada hace referencia a las capacidades dinámicas (dynamic capabilities) ([28], [30]). "Ésta determina la respuesta rápida y flexible a los cambios del entorno, y la capacidad de desarrollar nuevas capacidades y destruir las antiguas" [17]. Dentro de este grupo de factores la innovación en procesos y productos es un pilar fundamental.

Como resultados de la investigación en la industria chilena del salmón, mediante un análisis de correlaciones, Torres establece que los factores internos determinantes del éxito de proveedores-t en su incorporación a la industria son: "el empresario, a través de su educación y capacidad de gestión; la capacidad de crecimiento, a través de marketing, crédito a clientes y mejoramiento de infraestructura; la cooperación, en especial a través de joint ventures con empresas extranjeras como estrategia de entrada; y la capacitación de la mano de obra, factor importante para 
el cumplimiento de los requerimientos de calidad y precio de la industria" [31].

No obstante, las conclusiones obtenidas mediante el uso de correlaciones es limitada, dado que no permite medir la influencia del conjunto de variables exógenas sobre la variable endógena. Este artículo apunta precisamente a corroborar y complementar los resultados obtenidos por Torres, a través de un análisis econométrico.

Por otra parte, otras variables sugeridas por la teoría, como son la innovación y especialización asociada a cluster y aglomeraciones industriales, no han sido observadas en el estudio señalado como factores determinantes de éxito.

Respecto de la innovación, diversos autores hacen alusión a los proveedores como factor determinante de la competitividad de clusters, en particular, debido al traspaso de rentas económicas, nacidas de innovaciones consecutivas, a sus clientes por el proceso normal de derrame tecnológico y competencia ([19, 4, 10, 2, 6]). Schumpeter acuñó el término "innovación” aludiendo al real desarrollo de una idea a través de un proceso radical o de creación destructiva (de ideas anteriores) [27]. Esta definición ha continuado siendo elaborada llegando a distinguir distintos tipos de innovación: de producto (la introducción al mercado de un nuevo bien); de proceso (la introducción de un nuevo método de producción), de marketing (la apertura de un nuevo mercado o la implantación de una nueva estructura de mercado), de organización y de conquista de una nueva fuente de materias primas $([7,5])$. Cada uno de estos tipos concuerda con que la novedad es un criterio central y que las rentas económicas por sobre el promedio de los competidores es el resultado tangible de los procesos de innovación.

Recientemente se han rescatado diversas ideas plasmadas por autores en investigaciones previas para la evaluación de las características innovadoras de los proveedores, las cuales se pueden resumir en un modelo de siete proposiciones que determinan la evaluación de proveedores en el proceso de innovación de la empresa (cliente) [24]. Éstas se clasifican en tres grupos: características de los proveedores, características de la relación cliente-proveedor, y factores de soporte o facilitación. En particular las proposiciones relacionadas con las características de los proveedores innovadores son: las empresas especializadas son más innovadoras que aquellas empresas genéricas que abastecen a diversas industrias; las empresas con alta capacidad de desarrollo propia son probablemente más innovadoras; y los proveedores innovadores están envueltos en diversas acciones de colaboración al mismo tiempo.
Otro factor descrito para los clusters es la especialización productiva asociada al concepto de especialización flexible introducida en la obra "The second industrial divide" [18]. Los autores plantean la especialización flexible como una alternativa al Keynesianismo multinacional argumentando que las estructura industriales se organizarían considerando cuatro características: conglomeraciones regionales, empresas federadas, empresas solares y sus "orbitantes" proveedores de pequeño tamaño. En particular, esta última se refiere a la conjugación de una alta presencia de pequeñas y medianas empresas con el aprovechamiento de economías de escala externas ([18, 25]). Esto debido a la posibilidad de las pequeñas empresas de comparar tecnología y alcanzar una alta tasa de utilización de ella, dada la presencia de diversos potenciales clientes miembros de cluster. Esto conlleva a que los productores se especialicen tanto en el servicio al cluster como en la diversificación de productos de acuerdo a los cambiantes requerimientos.

\section{METODOLOGÍA Y ANÁLISIS DE RESULTADOS}

La determinación de los factores internos más importantes en el desarrollo de los proveedores-t es el resultado de una investigación empírica realizada entre noviembre del año 2003 y junio del año 2004, la cual incluyó 50 entrevistas a proveedores-t de diversos tipos (proveedores de manufacturas, de biotecnología y de servicios profesionales), de acuerdo a un universo de 300 empresas según el directorio de acuicultura y pesca 2002 [8]. El grupo objetivo de la investigación fue los proveedores de manufacturas (bienes de capital e insumos). La muestra (por conveniencia) tomada para el estudio consideró a 50 empresas localizadas en Santiago y Puerto Montt. Ésta incluyó empresas metalmecánicas, tales como fabricantes de maquinaria, balsas jaula, equipos e infraestructura; empresas no-metalmecánicas tales como, proveedores de redes, plásticos, fibra de vidrio y hormigón; y proveedores de insumos de la producción tales como guantes, material de empaque, insumos veterinarios y químicos. Los datos obtenidos en las entrevistas fueron procesados y analizados utilizando variables cualitativas ordinales e incorporándolas en un modelo econométrico de corte transversal, cuya metodología y resultados se pasan a describir a continuación.

Para el análisis del potencial interno de los proveedores el éxito como proveedor es representado por la variable endógena "cuota_referencia" que corresponde a la participación de mercado del proveedor en términos relativos respecto de la participación promedio de las empresas de cada sector (metalmecánico, no-metalmecánico 
y productores de insumos de la producción). Por otra parte, las variables exógenas que explican a la variable endógena están asociadas a la competitividad previa de la empresa antes de su inserción como proveedores y su estrategia de entrada ${ }^{3}$. La competitividad previa está compuesta por las variables: competitividad operacional, competitividad en investigación y desarrollo, competitividad en gestión, competitividad en finanzas y competitividad en marketing; mientras que la estrategia de entrada está asociada a la especialización de mercado de las empresas $\mathrm{y}$ acciones conducentes a posicionarse como proveedores de la industria salmonicultora ${ }^{4}$. El análisis considera un estudio de corte transversal para 50 empresas, considerando variables continuas y discretas (escala likert), como unidad de medida para las variables exógenas; y como unidad de medida para la explicada, una variable continua, las cuales aparecen en la tabla 1.

Una vez realizada la etapa de determinación de las variables relevantes en el caso de estudio, se procede a la etapa de especificación del modelo econométrico, a objeto de explicar el éxito de las empresas estudiadas en función del conjunto de variables exógenas determinadas. El modelo que se estudiará para la situación planteada es el siguiente:

CUOTA_REFERENCIA $=\mathrm{B}_{0}+\mathrm{B}_{1} *$ VAR5_1001 $+\mathrm{B}_{2} *$ VAR5_1101 + $\mathrm{B}_{3} * \mathrm{VAR} 5 \_701+\mathrm{B}_{4} * \mathrm{VAR} 6 \_1$ 301 + $\mathrm{B}_{5}$ *VAR6_2_301 + B 6 *VAR6_3_201 + B 7 *VAR6_5_101 $+\mathrm{B}_{8} *$ VAR6_6_501 $+\mathrm{B}_{9} *$ VAR6_2_201 $+\mu$

Mediante la estimación MCO (Mínimos Cuadrados Ordinarios $)^{5}$, se procede a la etapa de estimación de los parámetros de posición como de dispersión de cada uno de los estimadores, los cuales aparecen reflejados en la tabla 2 .

Por lo tanto el modelo queda definido de la siguiente manera:

CUOTA_REFERENCIA $=0.827 *$ VAR5_1001 + $20.899 *$ VAR5_1101+7.400*VAR5_701+ $1.286 *$ VAR6_1_301+1.219*VAR6_2_301 $+8.892 *$ VAR6_3_201 - 17.645*VAR6_5_101+ 30.668*VAR6_6_501 + 8.634*VAR6_2_201 - 194.395

3 Por lo tanto, la investigación incorporó preguntas retrospectivas para evaluar la competitividad de la empresa previa a su entrada como proveedor de la industria salmonicultora.

4 El modelo econométrico fue ejecutado con el software Eviews versión 4.1 .

5 Se usa la estimación MCO, pues cumple las propiedades deseadas para muestras finitas como asintóticas [33].
Tabla 1. Resultados de la aplicación del modelo de corte transversal.

Dependent Variable: CUOTA_REFERENCIA

Method: Least Squares

Date: 05/18/07 Time: 17:38

Sample: 150

Included observations: 50

\begin{tabular}{crlll}
\hline \hline Variable & Coefficient & Std. Error & t-Statistic & Prob. \\
\hline \hline VAR5_1001 & 0.827732 & 0.268510 & 3.082681 & 0.0037 \\
VAR5_1101 & 20.89963 & 7.621655 & 2.742137 & 0.0091 \\
VAR5_701 & 7.400217 & 5.187269 & 1.426611 & 0.1615 \\
VAR6_1_301 & 1.286373 & 0.430556 & 2.987703 & 0.0048 \\
VAR6_2_301 & 1.219602 & 0.513715 & 2.374083 & 0.0225 \\
VAR6_3_201 & 8.892485 & 8.770811 & 1.013873 & 0.3167 \\
VAR6_5_101 & -17.64538 & 8.527499 & -2.069233 & 0.0450 \\
VAR6_6_501 & 30.66823 & 11.79683 & 2.599701 & 0.0130 \\
VAR6_2_201 & 8.634017 & 6.568527 & 1.314453 & 0.1962 \\
& -194.3954 & 66.04972 & -2.943168 & 0.0054 \\
\hline \hline R-squared & 0.482449 & Mean dependent var & 99.96000 \\
Adjusted R-squared & 0.366000 & S.D. dependent var & 61.98056 \\
S.E. of regression & 49.35149 & Akaike info criterion & 10.81267 \\
Sum squared resid & 97422.79 & Schwarz criterion & 11.19507 \\
Log likelihood & -260.3167 & F-statistic & 4.143002 \\
Durbin-Watson stat & 1.857674 & Prob(F-statistic) & 0.000794 \\
\hline \hline
\end{tabular}

El coeficiente de determinación del modelo es de un $48 \%$, con un nivel de significatividad conjunta del $99 \%{ }^{6}$. En la tabla 2 es posible observar el valor de los coeficientes y de los niveles de significatividad individual para cada uno de los parámetros del modelo.

Para la etapa de validación se usaron los test de White ${ }^{7}$ para contrastar heteroscedasticidad, Durbin-Watson ${ }^{8}$ para autocorrelación y Jarque-Bera ${ }^{9}$ para verificar normalidad de los residuos. Todos los test aplicados validan las hipótesis del modelo econométrico planteado.

Los resultados mostrados en la tabla 2 indican que tanto las variables asociadas a la competitividad previa, como las asociadas a la estrategia de entrada de la empresa son determinantes del éxito como proveedores en términos de participación de mercado relativa a las empresas de su mismo sector.

Dentro de la estrategia de entrada de los proveedores destacan dos variables "cooperación horizontal" e "innovación en productos especializados". El coeficiente 7,4 para la primera, complementado con información recogida en las entrevistas nos lleva a concluir que aquellas empresas que cooperaron formalmente con sus pares han 
Tabla 2. Codificación y nombre de las variables.

\begin{tabular}{|l|l|l|}
\hline \multicolumn{1}{|c|}{ Código de Variables } & \multicolumn{1}{|c|}{ Variables } & \multicolumn{1}{c|}{ Indicadores } \\
\hline CUOTA_REFERENCIA & Participación de mercado & $\begin{array}{l}\text { Participación de mercado relativa a la participación } \\
\text { de mercado promedio de su sector }\end{array}$ \\
\hline VAR5_1001 & Especialización sectorial & \% de ventas destinada a la acuicultura \\
\hline VAR5_1101 & Innovación en productos especializados & $\begin{array}{l}\text { Variación reciente de cartera de productos para } \\
\text { la acuicultura }\end{array}$ \\
\hline VAR5_701 & Cooperación horizontal & $\begin{array}{l}\text { Cooperación horizontal: joint ventures, licencias } \\
\text { de fabricación y alianzas estratégicas }\end{array}$ \\
\hline VAR6_1_301 & Antigüedad de la empresa & Años de operación de la empresa \\
\hline VAR6_2_301 & Calificación de la mano de obra & $\begin{array}{l}\text { \% de empleados sólo con educación primaria } \\
\text { o secundaria }\end{array}$ \\
\hline VAR6_3_201 & Capacidad de innovación interna & Origen de las innovaciones \\
\hline VAR6_5_101 & Endeudamiento & Uso de crédito \\
\hline VAR6_6_501 & Capacidad para dar crédito a clientes & Disponibilidad para ofrecer crédito a clientes \\
\hline VAR6_2_201 & Diversificación de productos & Cartera de productos \\
\hline
\end{tabular}

logrado una mayor participación de mercado. La estrategia más común de cooperación ha sido el joint-venture con empresas extranjeras, lo cual abre el mercado a empresas extranjeras, pero a la vez permite a empresas locales fabricar conforme a diseños probados y con respaldo financiero, y, por lo tanto, en forma más competitiva. No obstante, este resultado debe ser considerado con cuidado, puesto que el nivel de significatividad es sólo de un $84 \%$. La segunda, en tanto, entrega un coeficiente 20,9 , lo cual significa que una variación entre $5 \%$ y $10 \%$ o entre $10 \%$ y $20 \%$, es decir un aumento al doble en la cartera de productos para la acuicultura, implica un aumento del $20,9 \%$ de la participación de mercado relativa, lo cual destaca la importancia de las capacidades dinámicas de los proveedores materializada a través de innovación en productos y la oferta de una amplia cartera de productos. Un tercer elemento de la estrategia de entrada es la "especialización sectorial". Aquí el resultado indica que la variación de un uno por ciento en el porcentaje de la concentración de las ventas en el mercado acuícola genera una variación de un 0,83\% en la participación de mercado relativa. Esto permite concluir que aquellas empresas que concentraron sus ventas en el mercado acuícola tendieron a lograr un mayor éxito de entrada como proveedor.

El factor más importante dentro de la competitividad previa de los proveedores ha sido la "capacidad de otorgar crédito a clientes". El coeficiente 30,1 refleja la ventaja de aquellas empresas con capacidad financiera para afrontar las inversiones requeridas por una demanda altamente creciente y, posteriormente, una concentración del mercado, implicando condiciones de pago más desfavorables. Esta variable es complementada con el coeficiente negativo $(-17,6)$ arrojado para la variable "endeudamiento", la cual implica que aquellas empresas que operaban con financiamiento propio tendieron a ser más exitosas que aquellas que operaban con créditos del sistema formal.

Otras dos variables relevantes son la "capacidad de innovación interna" y la "diversificación de productos" los cuales muestran coeficientes 8,9 y 8,6 respectivamente. El primero se refiere a que las empresas cuyas innovaciones en productos habían sido desarrolladas internamente han logrado una participación relativa de mercado superior en un $8,9 \%$ respecto a aquellas que han desarrollado las innovaciones en conjunto con el cliente, y estas últimas han logrado una participación 8,9\% mayor a aquellas en que las innovaciones han sido desarrolladas en forma externa. No obstante, estos valores deben ser interpretados con cuidado puesto que las variables tienen un nivel de significatividad de un $69 \%$ y un $80 \%$ respectivamente. La variable "antigüedad de la empresa" muestra un factor 1,3 , lo cual indica que cada año de antigüedad de la empresa genera $1,3 \%$ más de participación de mercado 


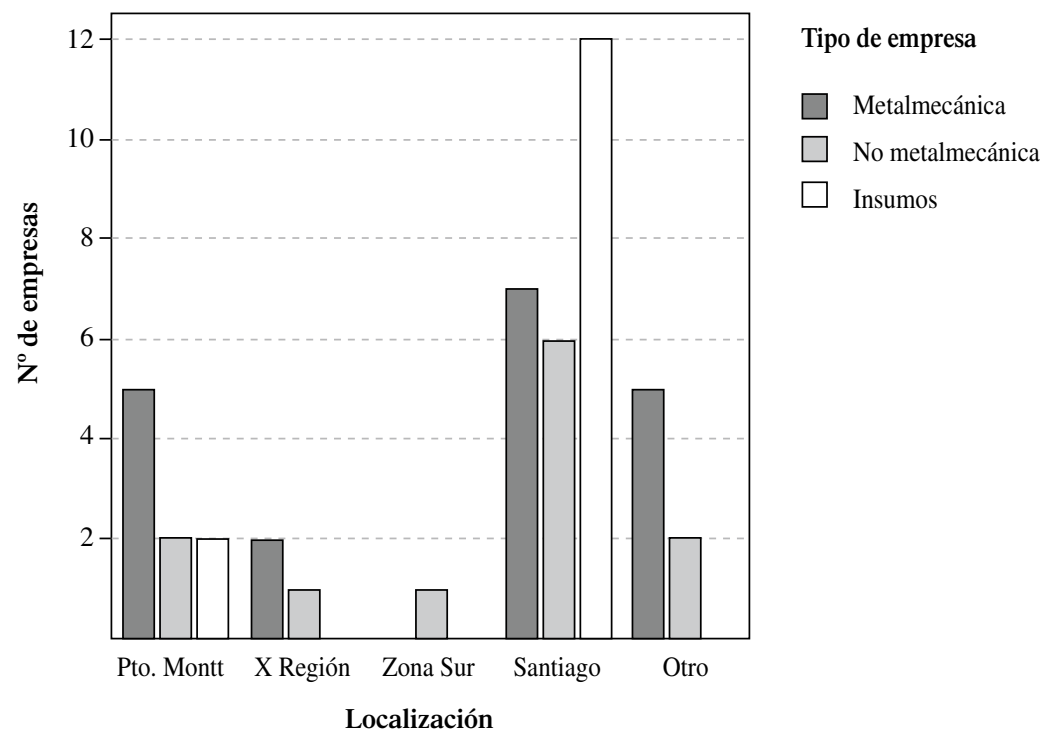

Figura 2. Ubicación de empresas por sector.

relativa. Es decir, aquellas empresas que se han incorporado como proveedores a través de un proceso de upgrading intersectorial han tendido a ser más exitosas que aquellas con menos años de operación. No obstante, debido a que el coeficiente es relativamente bajo, esta diferencia podría deberse a que las empresas más nuevas han alcanzado una participación relativa más pequeña del mercado en función de los pocos años de operación.

La variable "calificación de la mano de obra" muestra un coeficiente 1,2 , lo cual significa que una variación de un $1 \%$ en el porcentaje de mano de obra no calificada (es decir, sólo con educación primaria o secundaria) previo a la entrada como proveedor, genera un $1,2 \%$ más en la participación de mercado relativa. Si bien es cierto, el efecto en la variable endógena no es contundente, su efecto podría explicarse de dos maneras: la primera, es que las empresas con menos personal calificado realizaron inversiones en calificación de la mano de obra como estrategia de entrada; la segunda, que aquellas actividades que emplean alta mano de obra de baja calificación tendieron a lograr amplias participaciones de mercado. La primera conclusión se puede descartar, debido a que se descartó la significatividad de la variable "estrategia de capacitación", considerada en un modelo original. Mientras la segunda se puede corroborar al revisar las cuotas de mercado medias para los sectores no-metalmecánicos y productores de insumos, las cuales son considerablemente más altas que para los proveedores metalmecánicos. Los primeros grupos se caracterizan por ser actividades que emplean gran cantidad de mano de obra no calificada.
Aunque la localización de la empresa no ha sido determinante para el éxito de los proveedores, se puede agregar que los proveedores metalmecánicos tienden a estar ubicados en la Región de Los Lagos, mientras que los proveedores de insumos tienden a estar ubicados en Santiago, debido a que estos últimos son empresas afectas a importantes economías de escala y, por ende, necesitan estar cerca de mercados complementarios. Este efecto en la ubicación territorial se muestra en la figura 2.

\section{CONCLUSIONES}

El artículo revela la importancia de los proveedores en general y de los proveedores-t en particular dentro del contexto de la actual literatura asociada al concepto de cluster y cadena de valor. Dentro de este ámbito contribuye a abordar la brecha teórica relacionada con el estudio de proveedores intensivos en tecnología, que no son parte del eje central de la cadena de valor, respondiendo a la pregunta acerca de los principales factores internos que han determinado el éxito de proveedores locales de bienes manufacturados en el caso del cluster del salmón en Chile.

El artículo viene a corroborar parte de los resultados obtenidos por Torres [31], el cual propone una división entre factores asociados al potencial que ofrece la industria salmonicultora y los factores internos de los proveedores o potencial endógeno de los proveedores determinado por sus recursos (competitividad previa) y capacidades dinámicas (estrategia de entrada). 
El estudio se basa en un trabajo empírico para identificar los principales factores, a nivel de la empresa, que han determinado el éxito de los proveedores locales de manufacturas, en términos de participación de mercado. La investigación envolvió un levantamiento de material empírico a través de 50 entrevistas, las cuales fueron evaluadas cuantitativamente por medio de un análisis econométrico de corte transversal, usando variables explicativas ficticias.

En este sentido viene a profundizar los resultados obtenidos por Torres [31], quien sólo establece correlaciones entre variables, sin embargo, no determina el grado de influencia ni la influencia conjunta de las variables como determinantes de la participación de mercado.

Los resultados destacan la especialización sectorial, la innovación en productos especializados para la industria y la cooperación horizontal, dentro de la estrategia de entrada; y la antigüedad de la empresa, la capacidad de innovación interna, la capacidad financiera (capacidad de otorgar crédito a clientes y bajo nivel de endeudamiento) y la diversificación de la cartera productos, dentro de la competitividad previa, como determinantes del éxito de los proveedores.

Los resultados se contraponen a los presentados por Torres [31] que relevan la importancia del empresario, la capacitación de la mano de obra y el mejoramiento de la infraestructura. Por otra parte, corroboran los factores relacionados con cooperación horizontal y la capacidad de otorgar crédito a clientes.

Los resultados de este estudio, aunque corresponden a una aplicación particular, corroboran la importancia relevada por la teoría para los factores innovación y especialización productiva, elementos centrales de los proveedores integrados a un cluster.

\section{REFERENCIAS}

[1] A. Bigsten. "Relevance of the Nordic Model for African Development". UNU/WIDER Discussion Paper. $N^{\circ}$ 131. United Nations University, World Institute for Development Economics Research. 2001.

[2] P. Cooke. "Evolution of Regional Innovation Systems-Emergence, Theory, Challenge for Action”. En P. Cooke (eds.). Regional Innovation Systems. 2a Edición. Routledge. London. 2004.
[3] Directorio de Acuicultura y Pesca. Santiago: Technopress. 2002.

[4] D. Ernst. "New Challenges for Industrial Clusters and Districts: Global Production Networks and Knowledge Diffusion". En P. Guerrieri, S. Iammarino, C. Pietrobelli (eds.). The Global Challenge to Industrial Districts. Small and Medium-Sized Enterprises in Italy and Taiwan. Northampton: Edward Elgar. 2001.

[5] P. Escorsa Castells, J. Valls Pasola. "Manual de Gestión de Innovación Tecnológica en la Empresa". CINDA. Santiago, Chile. 1997.

[6] K. Esser. "Competitividad Sistémica. Competitividad Internacional de las Empresas y Políticas Requeridas". DIE Reports and Working Papers. $\mathrm{N}^{\circ} 11.1994$.

[7] J. Hauschildt. "Innovations management". $3^{\text {a }}$ Edición. München. 2004.

[8] H. Hernesniemi, M. Lammi and P. Ylä-Antilla. "Advantage Finland: The Future of Finnish Industries". P. Rouvinen (editor), ETLA - The Research Institute of the Finnish Economy. SITRA - The Finnish National Fund for Research and Development. Taloustieto Oy. Helsinki. 1996.

[9] J. Humphrey and H. Schmitz. "Developing Country Firms in the World Economy: Governance and Upgrading in Global Value Chains". INEF Report. $\mathrm{N}^{\mathrm{o}} 61$, Institut für Entwicklung und Frieden, Duisburg. 2002.

[10] R. Kaplinsky. "Globalisation and Unequalisation: What can be Learned from Value Chain Analysis?". Journal of Development Studies. Vol. $37 \mathrm{~N}^{\mathrm{o}} 2$, pp. 117-146. 2000.

[11] P. Krugman. "Geography and Trade". Cambridge, Massachusetts and London: The MIT Press. Leuven University Press. Leuven. 1991.

[12] J. Mahoney and J.R. Pandian. "The Resourcebased View within the Conversation of Strategic Management". Strategic Management Journal. Vol. 13, pp. 363-380. 1992.

[13] W.F. Maloney. "Missed Opportunities: Innovation and Resource-based Growth in Latin America". World Bank Policy Research Working Paper No 2935. 2002. 
[14] A. Marshall. "Principles of Economics". London and New York: Macmillan and Co. 1980.

[15] P. Maskell, A. Malmberg. "Localised Learning and Industrial Competitiveness". Cambridge Journal of Economics. Vol. 23, pp. 167-185. 2002.

[16] C. Montero. "Formación y Desarrollo de un Cluster Globalizado: el Caso de la Industria del Salmón en Chile”. ECLAC Series Productive Development. No 145. ECLAC. Santiago, Chile. 2004.

[17] E. Penrose. "The Theory of the Growth of the Firm”. New York: John Wiley. 1959.

[18] M. Piore and Ch. Sabel. "The Second Industrial Divide: Possibilities for Prosperity”. USA: Basic Books. 1984.

[19] M. Porter. "The Competitive Advantage of Nations". The Free Press. New York. 1990.

[20] F. Pyke and W. Sengenberger. "Industrial Districts and Local Economic Generation”. ILO. Geneva. 1993.

[21] J. Ramos. "Complejos Productivos en Torno a los Recursos Naturales: ¿Una Estrategia Prometedora?” In ECLAC Books. N 61. ECLAC. Santiago, Chile. 2001.

[22] R. Rumelt, D. Schendel and D. Teece. "Strategic Management and Economics". Strategic Management Journal. Vol. 12, pp. 5-29. 1991.

[23] "Salmonchile". Asociación gremial Salmonchile A.G. 2004.

[24] H. Schiele. "How to Distinguish Innovative Suppliers? Identifying Innovative Suppliers as New Task for Purchasing”. Industrial Marketing Management. Vol. 35, pp. 925-935. 2006.
[25] H. Schmitz. "Collective Efficiency and Increasing Returns". Cambridge Journal of Economics. Vol. 23, pp. 465-483. 1999.

[26] H. Schmitz and K. Nadvi. "Clustering and Industrialization: Introduction”. World Development. Vol. $27 \mathrm{~N}^{\circ}$ 9, pp. 1503-1514. 1999.

[27] J. Schumpeter. "The Theory of Economic Development”. Harvard University Press. 1934.

[28] J. Schumpeter. "Theorie der Wirtschaftlichen Entwicklung. Eine Untersuchung über Unternehmergewinn, Kapital, Kredit, Zins und den Konjunkturzyklus”. 7th Edition, Berlin: 1987: Duncker \& Humblot. 1911.

[29] P. Stevens. "Resource Impact-Curse or Blessing? A Literature Survey”. IPIECA. Vol. 13. Article 13-14. University of Dundee, Dundee. 2003.

[30] D. Teece and G. Pisano. "The Dynamic Capabilities of Firms: An Introduction”. Industrial and Corporate Change. Vol. 3 No 3, pp. 537-556. 1994.

[31] C. Torres. "Desarrollo de Proveedores en la Salmonicultura Chilena". Journal of Technology Management and Innovation. Vol. $2 \mathrm{~N}^{\circ}$ 1, pp. 92-107. 2007.

[32] B. Wernerfelt. "A Resource-based View of the firm”. Strategic Management Journal. Vol. 5 No 2. Autumn, pp. 418-438. 1984.

[33] J.M. Wooldridge. "Introducción a la Econometría. Un Enfoque Moderno”. Thomson South-Western, Segunda Edición. Mason, Ohio. 2006.

[34] P. Wright, M.J. Kroll and J. Parnell. "Strategic Management Concept and Cases". Prentice-Hall, Inc. Englewood Cliffs, New Jersey. 1996. 